\title{
A DES Procedure Applied to a Wall-Mounted Hump
}

\author{
Radoslav Bozinoski ${ }^{1}$ and Roger L. Davis ${ }^{2}$ \\ ${ }^{1}$ Thermal/Fluid Science \& Engineering Department, Sandia National Laboratories, Livermore, CA 945501, USA \\ ${ }^{2}$ Mechanical and Aero Engineering Department, University of California at Davis, Davis, CA 95616, USA
}

Correspondence should be addressed to Radoslav Bozinoski, rbozinoski@gmail.com

Received 1 March 2012; Revised 21 June 2012; Accepted 5 July 2012

Academic Editor: Linda L. Vahala

Copyright (C) 2012 R. Bozinoski and R. L. Davis. This is an open access article distributed under the Creative Commons Attribution License, which permits unrestricted use, distribution, and reproduction in any medium, provided the original work is properly cited.

This paper describes a detached-eddy simulation (DES) for the flow over a wall-mounted hump. The Reynolds number based on the hump chord is $\operatorname{Re}_{c}=9.36 \times 10^{5}$ with an in-let Mach number of 0.1 . Solutions of the three-dimensional Reynoldsaveraged Navier-Stokes (RANS) procedure are obtained using the Wilcox $k-\omega$ equations. The DES results are obtained using the model presented by Bush and Mani and are compared with RANS solutions and experimental data from NASA's 2004 Computational Fluid Dynamics Validation on Synthetic Jets and Turbulent Separation Control Workshop. The DES procedure exhibited a three-dimensional flow structure in the wake, with a $13.65 \%$ shorter mean separation region compared to RANS and a mean reattachment length that is in good agreement with experimental measurements. DES predictions of the pressure coefficient in the separation region also exhibit good agreement with experiment and are more accurate than RANS predictions.

\section{Introduction}

Simulation of "steady" and "unsteady" flow of aerodynamic bodies has matured a great deal over the past decade. Aerodynamic performance and flow structures can be predicted with acceptable accuracy except in the complex flow regions of mixing and at off-design conditions near stall. In these regions, flow structures with multiple eddies that mingle and mix are often not predicted well due to inadequate computational grid density and a breakdown of turbulence models.

The focus of the current effort has been to validate the Navier-Stokes code and to further develop the detachededdy viscosity model using the wall-mounted hump grid. This case proved difficult for most of the participants of the NASA workshop, especially those using two or even threedimensional RANS techniques [1-3]. Most, if not all, participants using Reynolds-averaged Navier-Stokes predicted too large a separation region, which clearly showed the limitations presented with simply using a RANS procedure. Efforts by Morgan et al. [1] to surpass the limitations of RANS models with high-order spacial and temporal methods applied to the Navier-Stokes and $k-\epsilon$ equations showed little improvement, especially in the separation region of the flow. Similar results were obtained by Balakumar [3] using a fifth-order accurate weighted essentially nonoscillatory (WENO) scheme for space discretization and a third-order, total variation diminishing (TVD) Runge-Kutta scheme for time integration. Israel et al. [4] evaluated a direct numerical simulation (DNS) as well as a flow simulation methodology (FSM) where the RANS closure equations are combined with a contribution function that is used to determine the magnitude of the turbulent stress term. They observed good agreement between FSM, DNS, and the experimental data and suggest that differences between the numerical predictions and experiment can be attributed to discrepancies in the numerical representation of the experiment rather than deficiencies in the numerical methodology. Miller and Seitz [5] compared a SAS model proposed by Menter, Kuntz, and Bender [6] as well as DES and found that both the methods produced results in good agreement with experiment and prove to be more appropriate for industrial applications since they are less computationally intensive than DNS and LES simulation. 


\section{Governing Equations}

The unsteady, Favre-averaged governing flow-field equations for an ideal, compressible gas in the right-handed, Cartesian coordinate system using primary variables are used. The three-dimensional continuity, momentum, and energy equations can be written in conservative form as follows:

$$
\begin{gathered}
\frac{\partial \rho}{\partial t}+\frac{\partial\left(\rho u_{j}\right)}{\partial x_{j}}=0, \\
\frac{\partial \rho u_{i}}{\partial t}+\frac{\partial\left(\rho u_{j} u_{i}\right)}{\partial x_{j}}=-\frac{\partial P}{\partial x_{i}}+\frac{\partial \hat{\tau}_{i j}}{\partial j}, \\
\frac{\partial E}{\partial t}+\frac{\partial\left(\rho u_{j} H\right)}{\partial x_{j}}=\frac{\partial}{\partial x_{j}}\left[u_{i} \hat{\tau}_{i j}+\left(\frac{\mu}{\operatorname{Pr}}+\frac{\mu_{T}}{\operatorname{Pr}_{t}}\right) \frac{\partial h}{\partial x_{j}}\right], \\
\hat{\tau}_{i j}=\tau_{i j}+\tau_{i j}^{R}=\left(\mu+\mu_{T}\right) S_{i j}, \\
S_{i j}=\frac{1}{2}\left(\frac{\partial u_{i}}{\partial x_{j}}+\frac{\partial u_{j}}{\partial x_{i}}\right)-\frac{2}{3} \frac{\partial u_{k}}{\partial x_{k}} \delta_{i j} .
\end{gathered}
$$

Since we are dealing with compressible flows, we require an equation of state to relate the energy with pressure and enthalpy

$$
\begin{aligned}
E & =\rho\left[e+\frac{1}{2} V^{2}\right], \\
H=h+\frac{1}{2} V^{2} & =\frac{\gamma}{\gamma-1} \frac{P}{\rho}+\frac{1}{2} V^{2}=\frac{E+P}{\rho} .
\end{aligned}
$$

Additional governing equations as developed by Wilcox $[7,8]$ are used for the transport of turbulent kinetic energy and turbulence dissipation rate in regions of the flow where the computational grid or global time-step size cannot resolve the turbulent eddies. The equations for the turbulent mixing energy and specific dissipation rate can be seen below and are Wilcox 2006 [7] model

$$
\begin{gathered}
\mu_{T}=\frac{\rho k}{\omega}, \\
\frac{\partial \rho k}{\partial t}+\frac{\partial \rho u_{j} k}{\partial x_{j}}=\tau_{i j}^{R} \frac{\partial u_{i}}{\partial x_{j}}-\beta_{k} \rho \omega k+\frac{\partial}{\partial x_{j}}\left[\left(\mu+\sigma^{*} \mu_{T}\right) \frac{\partial k}{\partial x_{j}}\right], \\
\frac{\partial \rho \omega}{\partial t}+\frac{\partial \rho u_{j} \omega}{\partial x_{j}}=\left(\frac{\gamma \omega}{k}\right) \tau_{i j}^{R} \frac{\partial u_{i}}{\partial x_{j}}-\beta_{\omega} \rho \omega^{2}+\frac{\partial}{\partial x_{j}}\left[\left(\mu+\sigma \mu_{T}\right) \frac{\partial \omega}{\partial x_{j}}\right],
\end{gathered}
$$

where

$$
\begin{array}{cc}
\beta_{k}=\frac{9}{100}, & \beta_{\omega}=\frac{3}{40}, \\
\sigma^{*}=\frac{1}{2}, & \sigma=\frac{1}{2} .
\end{array}
$$

In regions of the flow where the larger-scale eddies can be resolved with the computational grid, techniques borrowed from large-eddy simulation are used to represent the viscous shear and turbulent viscosity. The large-eddy subgrid model described by Smagorinsky [9] is modified according to the detached-eddy considerations described by Strelets [10] and Bush and Mani [11]

$$
\mu_{T}=\rho l_{l e} \sqrt{k}
$$

where $l_{l e}$ is an eddy length scale proportional to the grid/time-step filter width, $\Delta$ :

$$
l_{l e}=\min \left(\frac{\sqrt{k}}{\omega}, \beta_{k} C_{\mathrm{des}} \Delta\right) .
$$

In addition, the dissipation term, $\beta_{k} \rho k \omega$, of the turbulent kinetic energy transport equation (4) is limited by the eddy length scale, $l_{l}$, according to

$$
\beta_{k} \rho k \omega \Longrightarrow \beta_{k} \rho k * \max \left(\omega, \frac{\sqrt{k}}{\beta_{k} C_{\mathrm{des}} \Delta}\right),
$$

where $C_{\text {des }}$ is a proportionality coefficient

$$
\Delta=\max (d x, d y, d z, u * d t, \sqrt{k} * d t) .
$$

Equation (10) determines the smallest eddies that can be resolved. The first three terms determine what the minimum eddy size the grid can support. The two time terms are the convection velocity and subgrid scale turbulence, respectively. They are included to ensure that the time step is small enough to resolve the unsteady effects. Equation (7) can be made to resemble (3) using the following:

$$
\begin{gathered}
\omega_{B}=\max \left(\omega, \frac{\sqrt{k}}{\beta_{k} C_{\mathrm{des}} \Delta}\right), \\
\mu_{T}=\frac{\rho k}{\omega_{B}} .
\end{gathered}
$$

\section{Numerical Techniques}

The conservation of mass, momentum, and energy equations are solved using a Lax-Wendroff control-volume, timemarching scheme as developed by $\mathrm{Ni}$ [12], Dannenhoffer [13], and Davis et al. [14, 15]. Numerical solutions of unsteady flows can be performed with either the explicit [12] or a dual time-step procedure [16]. These techniques are second-order accurate in time and space. A multiplegrid convergence acceleration scheme [12] is used for steady, Reynolds-averaged Navier-Stokes solutions and the inner convergence loop of unsteady simulations using the dual time-step scheme. The approach is called MBFLO and has two-dimensional [17], axi-symmetric [18] (with and without swirl), and three-dimensional [19] versions. The three-dimensional procedure and results for a DES using the Bush and Mani algorithm for flow over a wall-mounted hump are described here.

The combined second- and fourth-difference dissipation model of Jameson [20] is used in the current procedure for both the mean flow and turbulence equations. The 
TABLE 1: Typical speed-up and efficiencies.

\begin{tabular}{lccc}
\hline Processes & Speed Up & Efficiency & Pts/Process \\
\hline 1 & 1.00 & $100 \%$ & $1,780,440$ \\
2 & 2.00 & $100 \%$ & 892,440 \\
4 & 4.00 & $100 \%$ & 448,440 \\
8 & 7.79 & $97 \%$ & 226,440 \\
16 & 14.60 & $91 \%$ & 115,440 \\
20 & 18.06 & $90 \%$ & 93,240 \\
40 & 31.39 & $78 \%$ & 48,840 \\
\hline
\end{tabular}

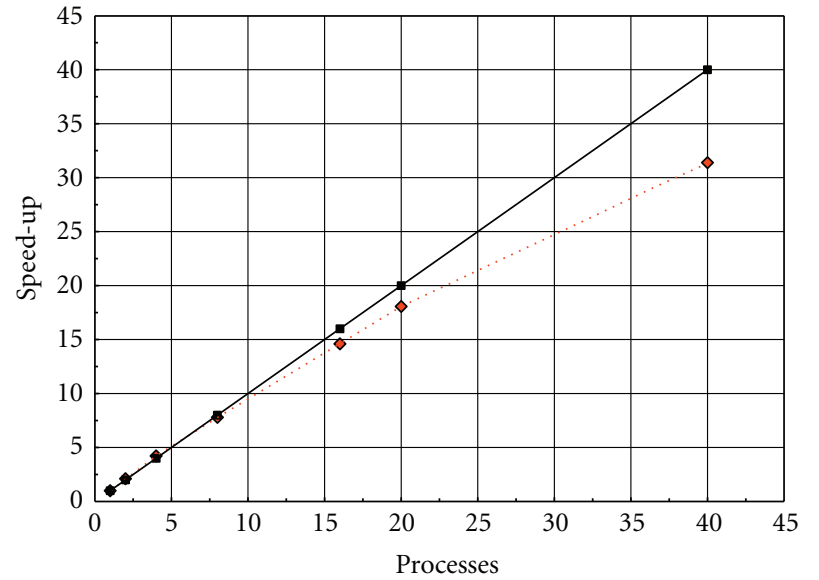

$\rightarrow$ Linear speed-up

$\diamond$ Actual speed-up

Figure 1: Typical Speed-Up factor as a function of number of processes.

fourth-difference dissipation is scaled by the inverse of the absolute value of the mean strain rate squared. This function decays the numerical dissipation in all viscous flow regions, including boundary layers, wakes, large eddies, and secondary flows.

Parallelization is performed using the Message Passing Interface (MPI) library [21]. Figure 1 and Table 1 show the typical speed-up and associated efficiencies as functions of the number of processors. The configuration used to generate this data was similar to that shown below in the results section where the computational grid consisted of 1.80 million grid points. The data was generated on a Linux cluster consisting of $3.6 \mathrm{GHz}$ Intel Xeon processors. Figure 1 shows that a speed-up factor of 18.06 is realized with 20 processes yielding a 90\% parallel efficiency. In Table 1, we can see that efficiencies of $90 \%$ and higher can be obtained if no less than 100, 000 grid points per process are used.

\section{Results}

The Navier-Stokes codes has been verified with analytical data for a series of standard test cases such as steady inviscid flow over circular bumps, turbulent flow over airfoils, and laminar and turbulent flow over a flat plate, as well as axi-symmetric flows [17-19]. The focus of the current investigation is to demonstrate and validate the DES model for a separated flow and to determine what advantage may exist, in terms of accuracy, for DES compared to threedimensional URANS and its applicability in the design process.

The simulation of a turbulent flat plate, constructed in such a way as to develop the inflow boundary conditions for the wall-mounted hump, was initially performed. The length of the flat plate was determined through preliminary CFD tests, so the predicted boundary layer thickness was essentially that of the experiment. The flow conditions were also set to match the wall-mounted hump with $\mathrm{Ma}=0.1$ and $\operatorname{Re}_{c}=9.36 \times 10^{5}$.

For the flat plate mesh, the computational domain extended from $-2.80 \leq x / c \leq 4.25$ in the streamwise direction and from $0.0 \leq y / c \leq 0.909$ in the normal direction. There were 121 points used in the streamwise direction with a maximum stretching ratio of $\Delta x=1.2$. The wall spacing normal to the surface was set to $3.6 \times 10^{-6}$ and corresponds to $\Delta y^{+}=0.25$. A maximum stretching ratio of 1.2 was achieved with 177 points clustered near the flat plate surface.

The simulation was performed by initializing the inlet with a uniform flow field where the nondimensional velocity components were set to $u=1, v=0$, and $w=0$ and nondimensional density to $\rho=1$. In the area over the flat plate, the velocity profile was initialized using the log-law profile, and density and energy were recomputed to keep the pressure at the freestream. During the computation, the inflow boundary condition held total pressure and total temperature constant, while at the exit, static pressure was held constant. Along the flat plate surface from the inlet to the leading edge of the flat plate, an inviscid wall boundary condition was imposed with an adiabatic no-slip wall over the entire flat plate surface. The upper wall of the flow domain was also set to an inviscid wall.

To verify the predicted solution, the velocity profile was plotted in terms of the inner layer velocity and length variables $u^{+}$and $y^{+}$, respectively, and compared to the empirical viscous sublayer, log-law, and 1/7th power law relations as shown in Figure 2(a). Figure 2(b) shows the predicted velocity and experimentally measured profiles at $x / c=-2.8$, which corresponds to the inlet of the wallmounted hump.

Once the inlet conditions were generated using the flat plate, the wall-mounted hump test case for turbulent flow 


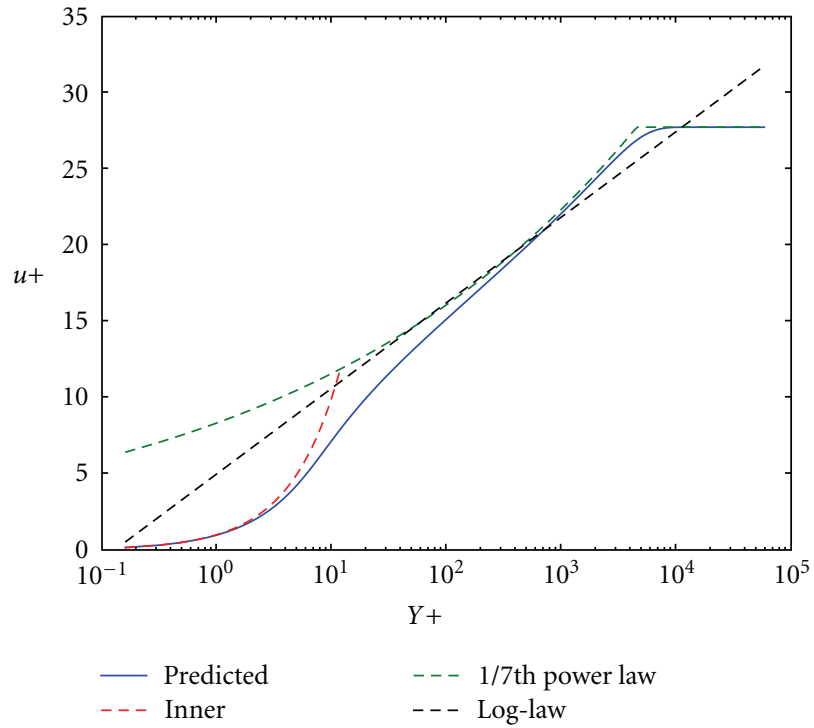

(a)

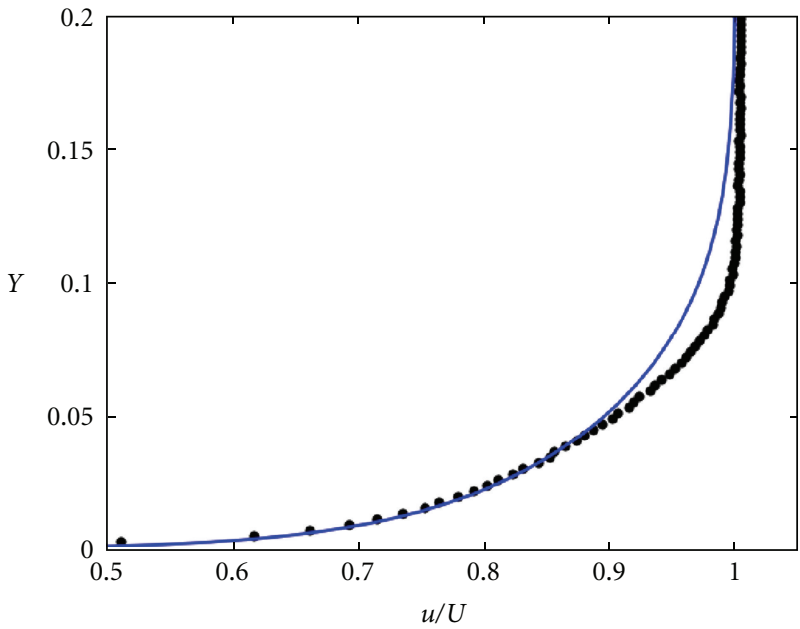

- Experiment Predicted

(b)

FIGURE 2: (a) Inflow $U$-velocity profile in inner-wall coordinates. (b) A turbulent RANS $U$-velocity profile comparison.

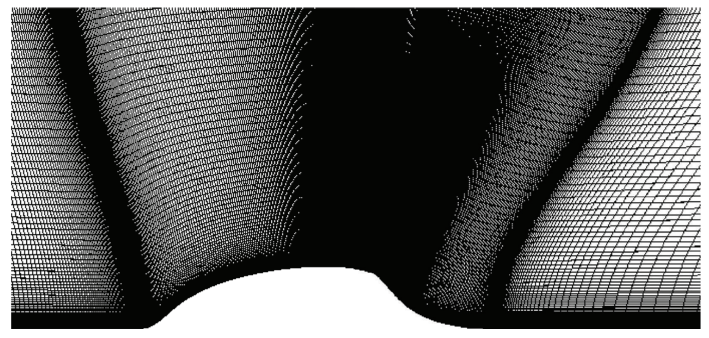

FIGURE 3: Computational grid for wall-mounted Hump (note: every fifth point is shown).

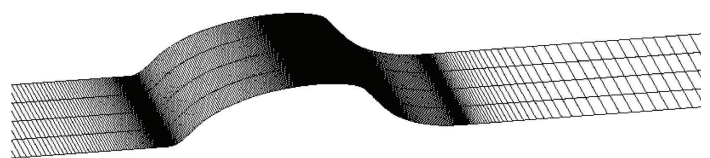

FIGURE 4: Surface grid for 3D unsteady cases (note: 5 spanwise planes are shown).

was simulated and compared to experimental data from the NASA workshop [22] that focused on synthetic jets and turbulent separation control. The hump geometry was constructed to simulate a $20 \%$ thick Glauert-Goldschmied airfoil with a chord length of $0.472 \mathrm{~m}(1.38 \mathrm{ft}$.), a maximum height of $0.054 \mathrm{~m}(0.176 \mathrm{ft}$.), and a span of $0.584 \mathrm{~m}(1.92 \mathrm{ft}$.$) .$ The experimental data was obtained for $\mathrm{Ma}_{\infty}=0.10$ and $\operatorname{Re}_{c}=9.36 \times 10^{5}$ based on the chord. The test case that was investigated was without flow control, where there was no blowing or suction in the slot. The numerical results obtained used a three-dimensional structured grid smoothed over the slot and with the top wall shape adjusted to approximately account for side plate blockage, as recommended by the workshop [22]. The grid shown in Figure 3 extended

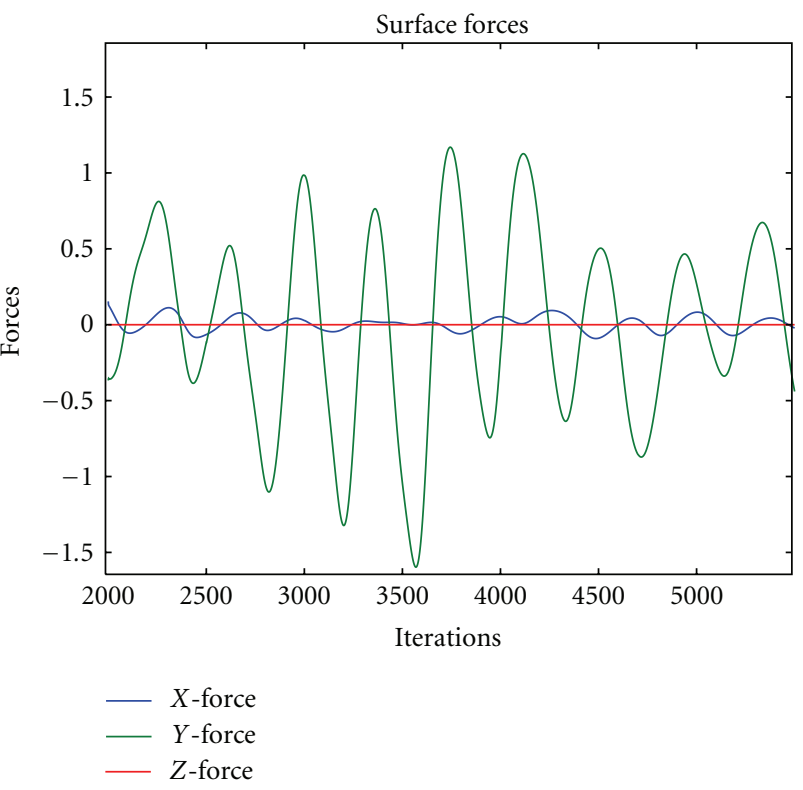

Figure 5: Lift and drag signals for three-dimensional simulation.

from $x / c=-9.20$ to 3.5 in the streamwise direction, with the hump located from $x / c=0.0$ to 1.0 and from $y / c=0.0$ to 0.9 in the normal direction. The mesh contained 155, 937 grid points with 881 in the streamwise direction and 177 normal to the wall. An initial RANS simulation was performed using three planes in the spanwise direction for a grid that consisted of 467,811 points. This allowed a nominally twodimensional flow simulation to be performed and compared to the experiment. For true three-dimensional simulations, the original $881 \times 177$ grid described was used and extruded in the spanwise direction 0.15 chord lengths and can be seen 


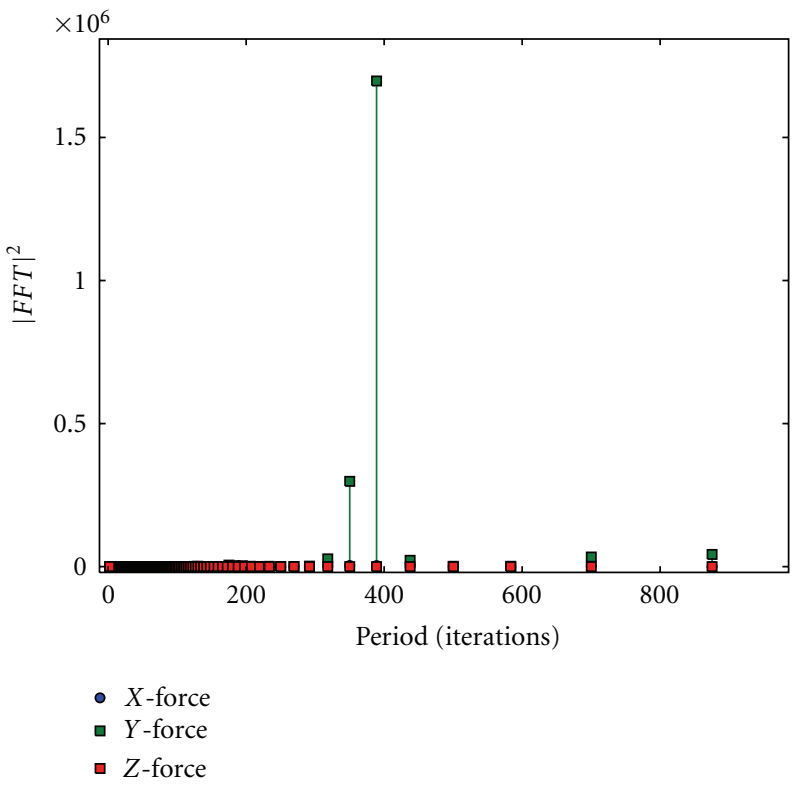

(a) DES PSD

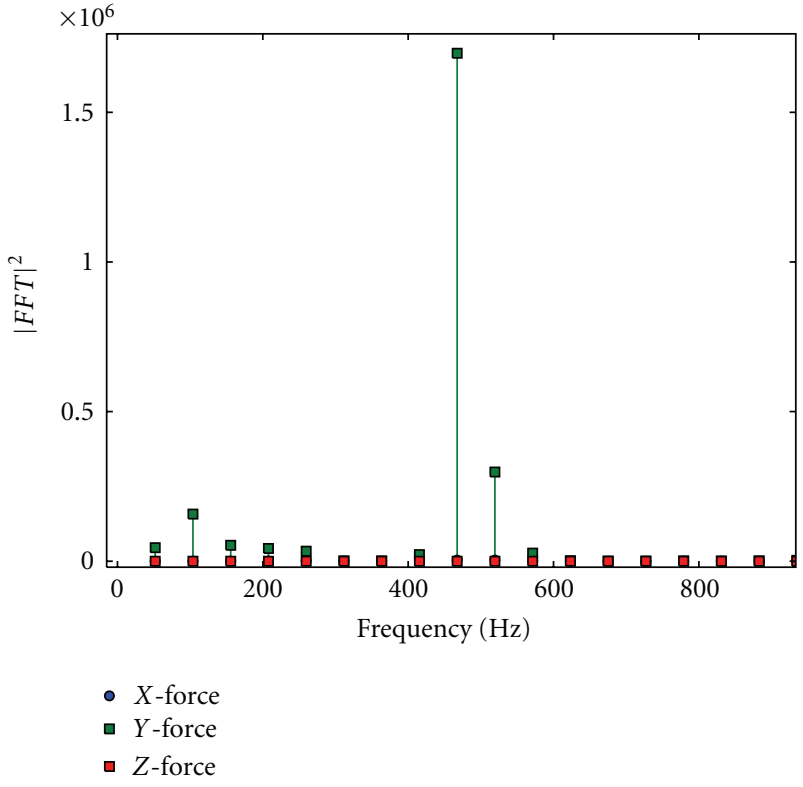

(b) DES PSD (Hz)

FIgURE 6: Lift and drag signals for three-dimensional simulation.

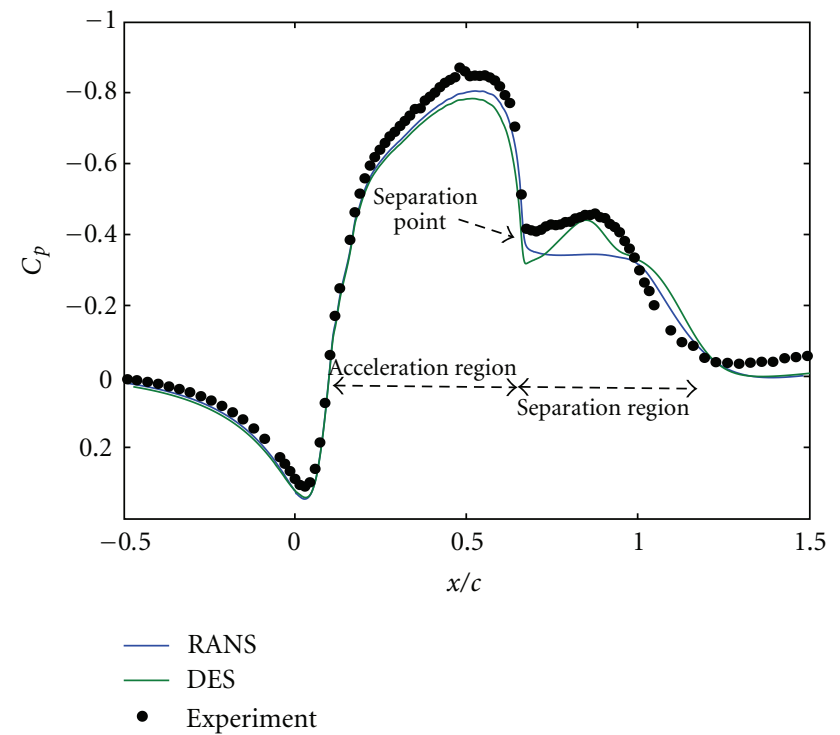

Figure 7: Pressure coefficient comparison.

in Figure 4. The spanwise direction was meshed using 49 points with uniform spacing and corresponds to roughly two boundary layer thicknesses based on the inlet.

With the aid of the turbulent flat plate simulation, the inflow velocity and density profiles for the wall-mounted hump case were generated and used to initialize the flow domain over the wall-mounted hump and helped decrease the computational time required to obtain a solution. The top of the flow domain was set with an inviscid wall boundary condition with an adiabatic no-slip wall along the south boundary starting at the inlet and leading over the hump section. Static pressure was once again held constant at the exit of the flow domain. For the nominally twodimensional case using the steady RANS solver, inviscid walls were imposed for the spanwise boundaries, whereas periodic boundary conditions were used for the three-dimensional unsteady simulation. The turbulent freestream intensity, Tu, and the dissipation length scale, $l_{\text {dis }}$, were set to $1.00 \%$ and 0.06 , respectively. The DES coefficient used was that suggested by the original authors of a value of 0.6 [11].

The temporal periodicity and unsteady behavior was initially studied for the three-dimensional DES case. The information obtained was then used to determine the number of time-steps necessary to resolve a minimum of 10 periodic cycles for the time-averaged DES at the given global time-step. Figures 5 and 6 (a) show the signal history for the instantaneous $x$-, $y$-, and, $z$-surface forces as well as the Power Spectral Density (PSD) as a function of the number of time-steps. The PSD of surface forces was plotted here as a function of the number of time-steps to more readily see periodicity in the flow. In Figure 6(a), we see that the peak spectral signal is repeated approximately every 400 time-steps, which corresponds to a frequency of $420 \mathrm{~Hz}$. The corresponding Strouhal number based on the hump height and freestream speed is approximately 0.65 . Once the period was determined, this case was run for approximately 10 periodic cycles and time-averaged.

Figure 7 shows the time-averaged pressure coefficient distribution resulting from the RANS and time-averaged DES simulations. The numerical results predicted using the RANS procedure produced higher pressure levels in the separation region, $0.65 \leq x / c \leq 1.3$, when compared to the experimental data. This is consistent when compared to other RANS simulations [1-3] in the NASA workshop. 


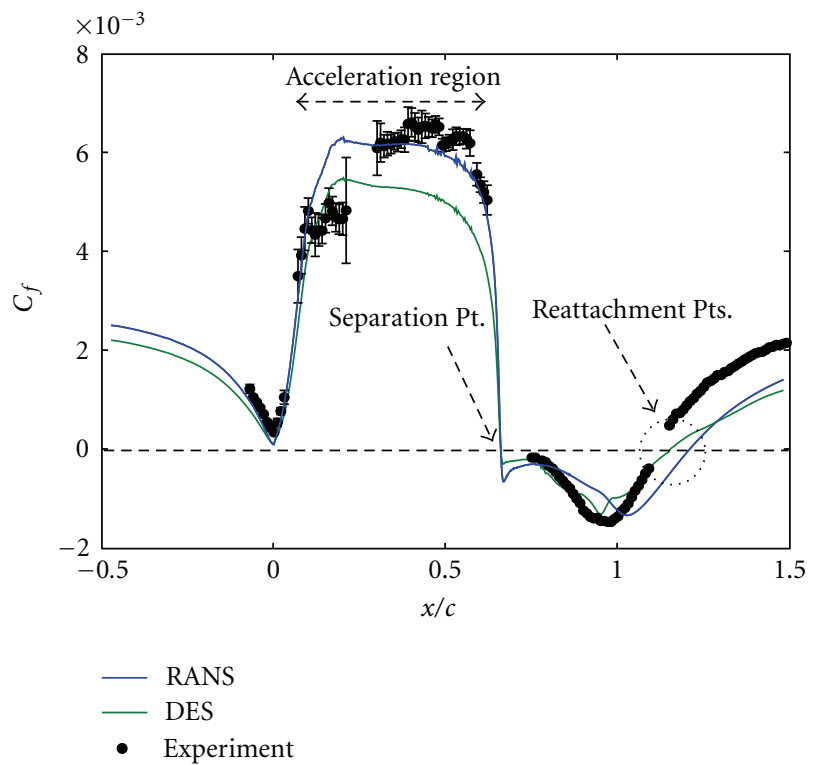

(a) RANS and DES

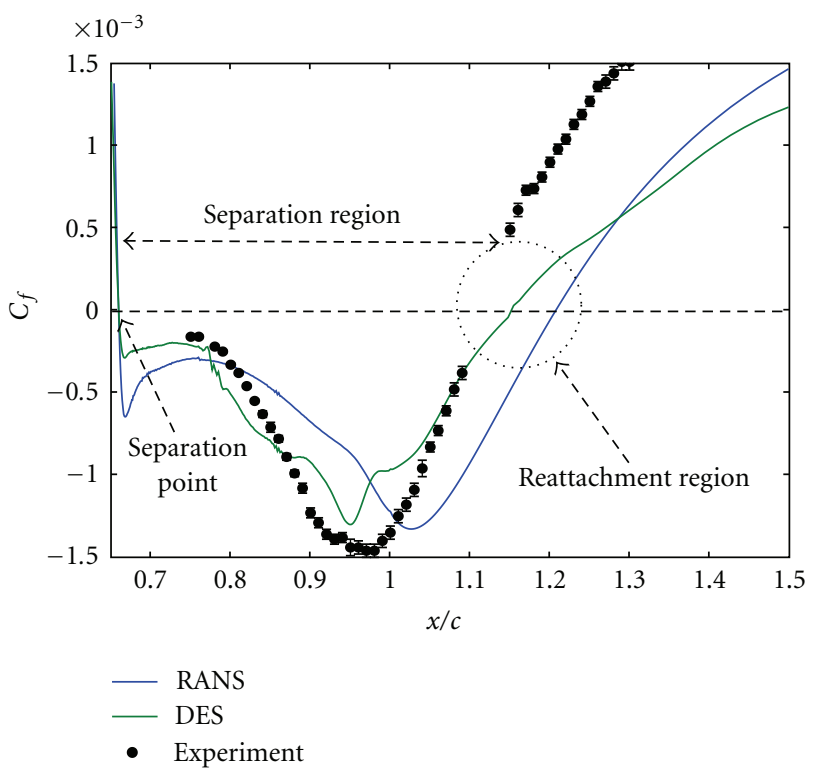

(b) DES in separation region

FIgURE 8: Predicted skin friction of time-averaged RANS and DES.

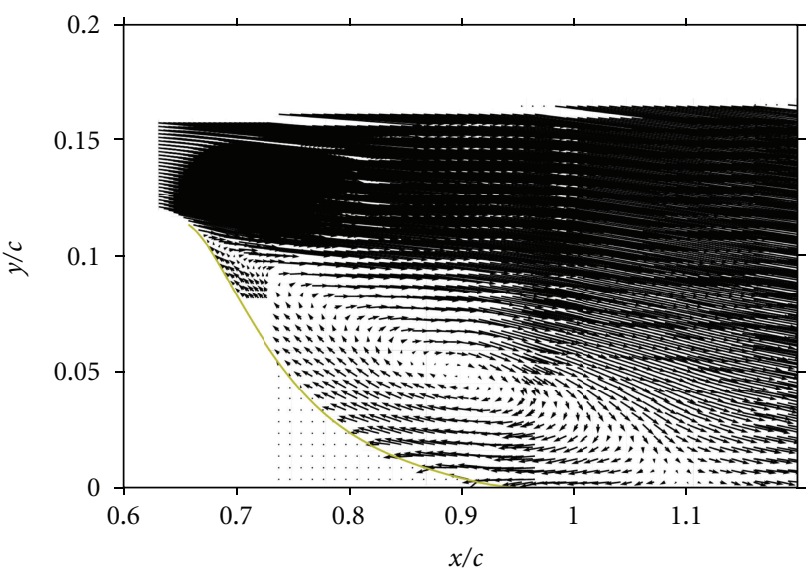

(a) Experimental PIV

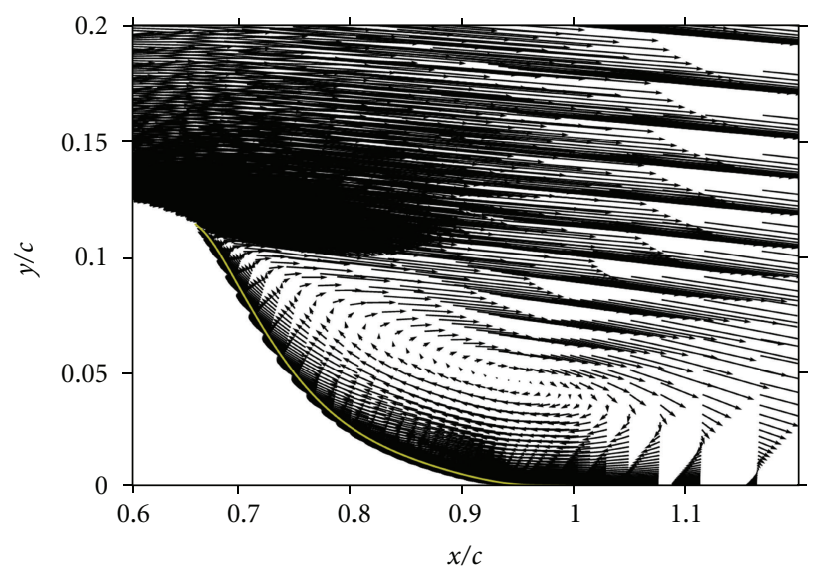

(b) RANS

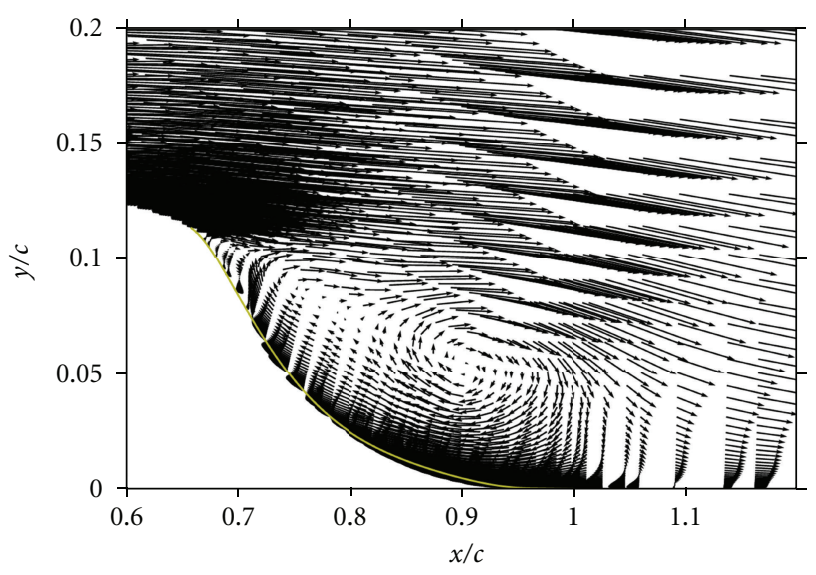

(c) Time-averaged DES

Figure 9: Comparison of $U$-velocity contours. 


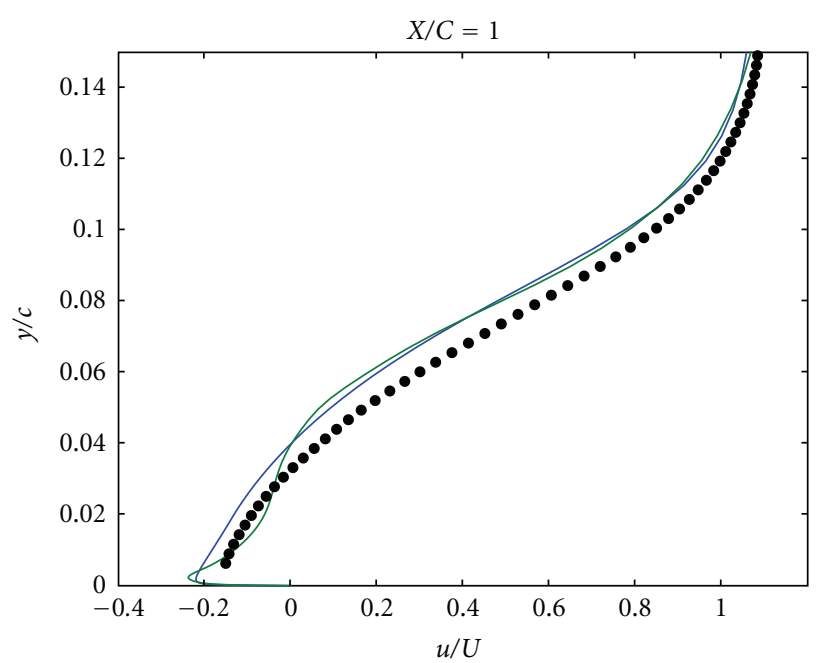

(a)

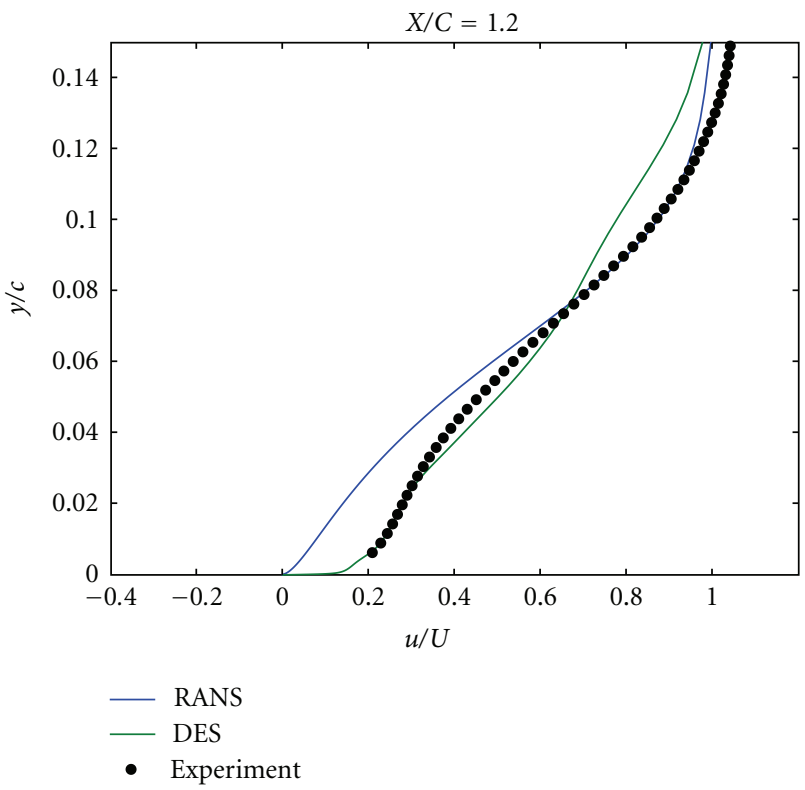

(c)

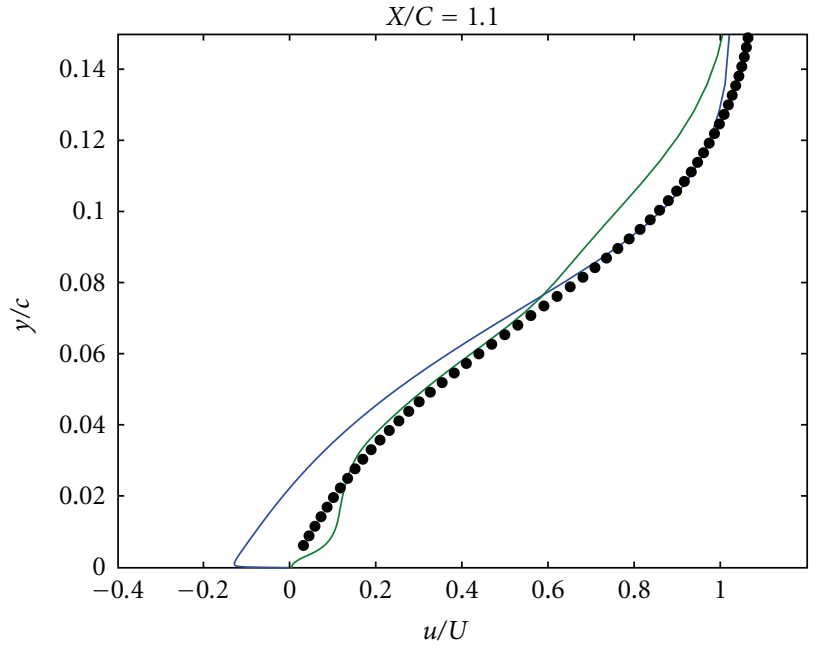

(b)

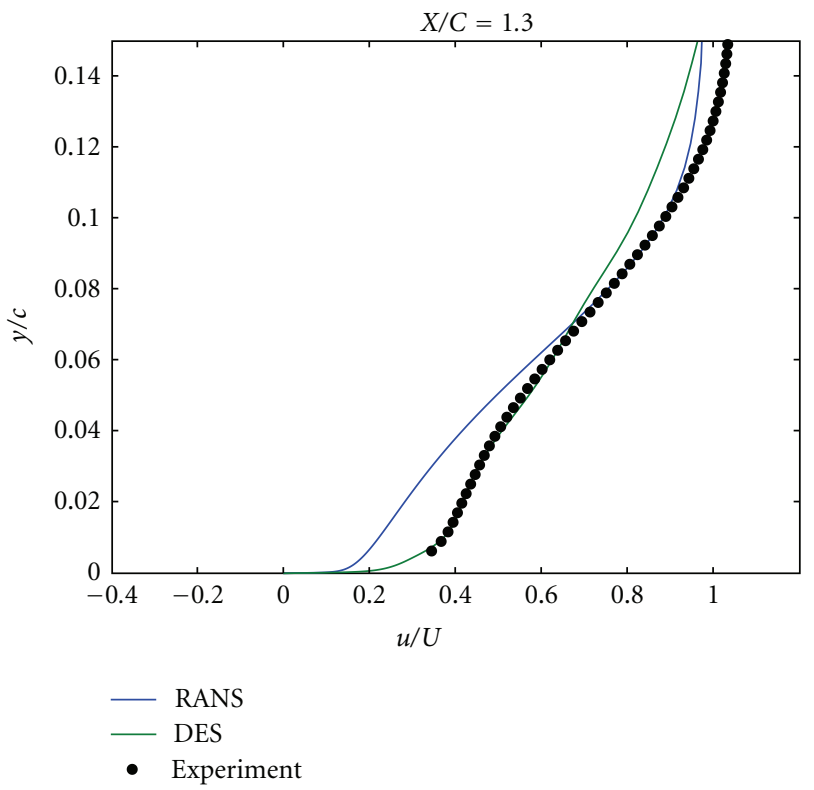

(d)

Figure 10: Mean $U$-velocity profile comparison at $x=$ (a) 1.0, (b) 1.1, (c) 1.2, and (d) 1.3 .

Researchers who used shear-stress transport (SST) RANS models showed similar predictions for the pressure leading up to the hump as well as higher pressures in the separation region. The baseline RANS cases run by Morgan, Rizzetta, and Visbal [1] also showed similar pressure predictions for the flow leading up to the hump and in the separation region. RANS simulations run by Krishnan, Squires, and Forsythe [2] also showed similar predictions as well as those made by Balakumar [3] and Šarić et al. [23]. The current DES case showed similar comparison with the experimental data in the acceleration region of the flow, $0.00 \leq x / c \leq 0.65$, with significant improvement in the separation region located between $0.65 \leq x / c \leq 1.3$.

A comparison of the time-averaged skin-friction coefficient, $C_{f}$, is shown in Figure 8. Once again, there is good agreement with the steady RANS prediction upstream of the hump to $x / c=0$ and over the hump to the beginning of the separation region $x / c=0.65$. The DES case slightly underpredicts the skin friction in the acceleration region of the flow with a max value of approximately 0.005 . Similar results were shown by Morgan et al. [24] using an implicit large-eddy simulation. Both simulations accurately predicts the onset of the separation region at approximately $x / c=0.65$. In the separation region, we see the predicted reattachment point for the RANS case is at $x / c=1.2$ and the time-averaged DES at $x / c=1.105$. The DES matches the experimental data in the separation region much better than the RANS procedure. Table 2 shows an overview of the separation and reattachment locations for the RANS and DES procedures compared to the experimental data. Here, 


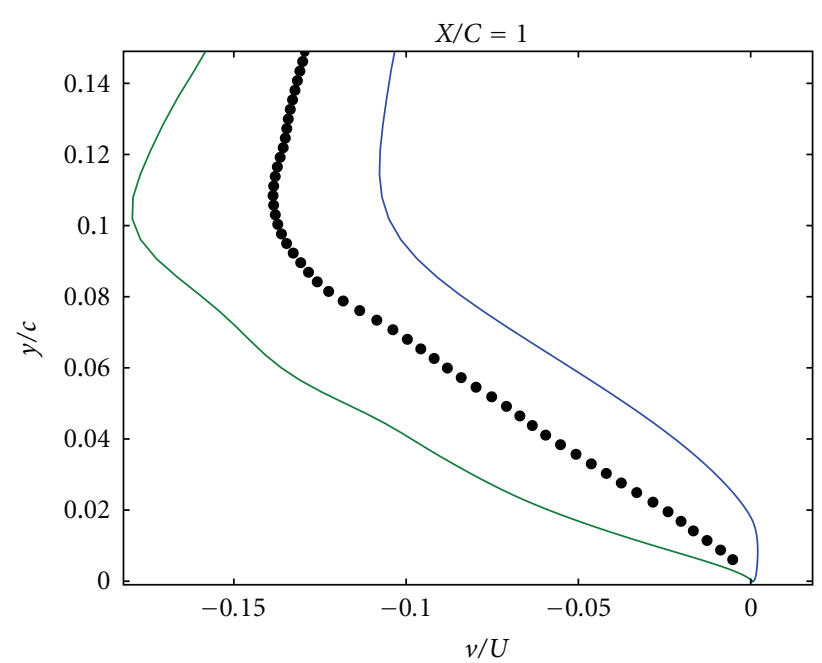

(a)

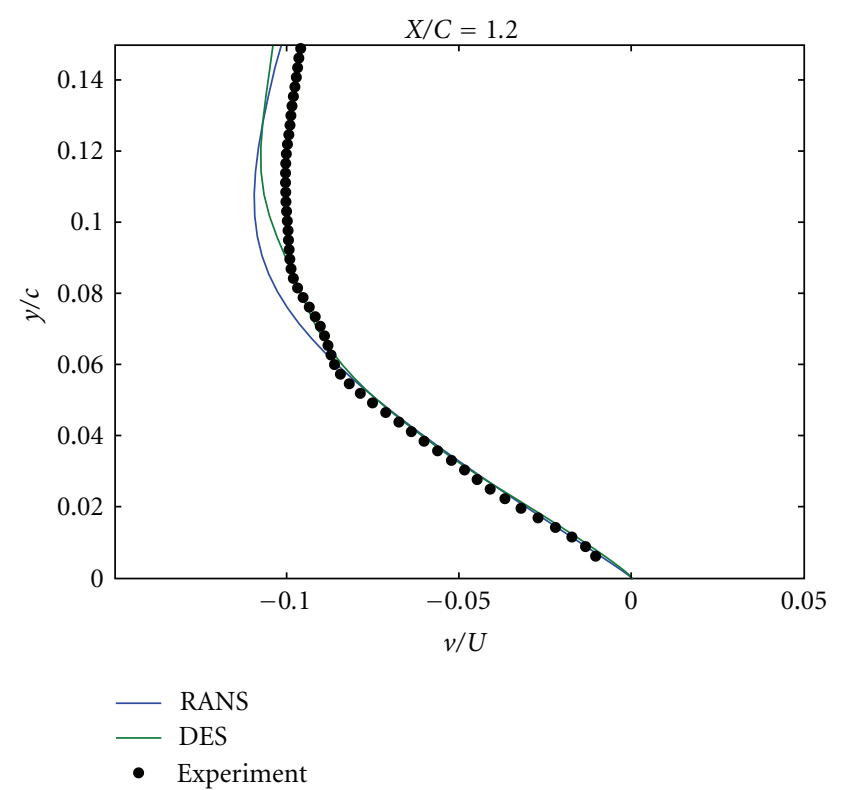

(c)

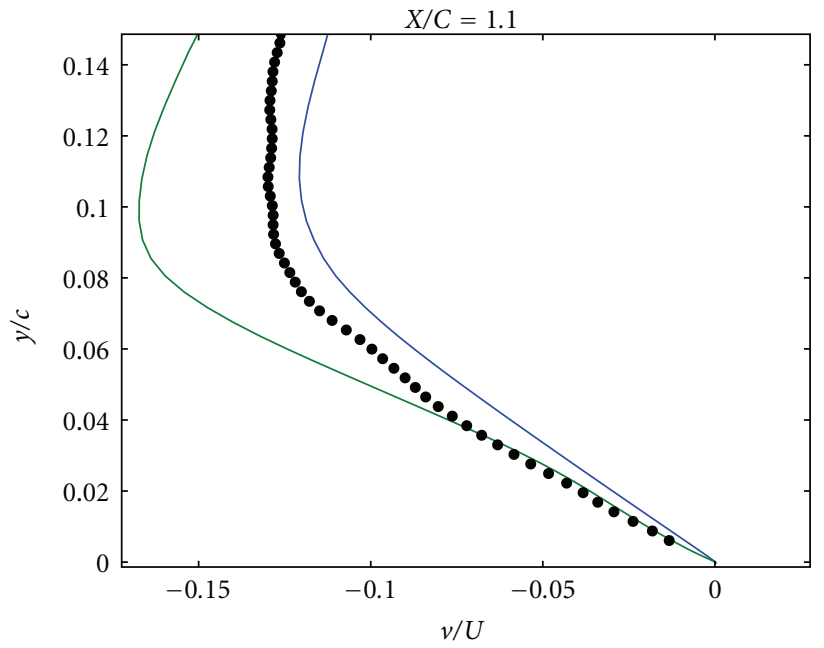

(b)

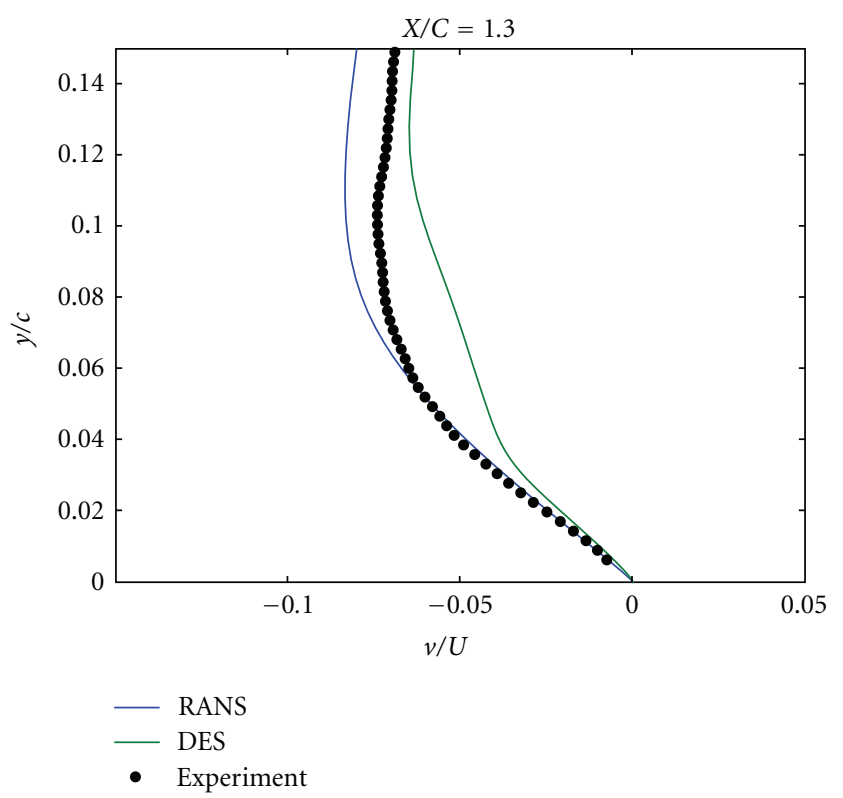

(d)

Figure 11: Mean $V$-velocity profile comparison at $x=$ (a) 1.0, (b) 1.1, (c) 1.2, and (d) 1.3.

TABLE 2: RANS and DES Comparison.

\begin{tabular}{lcc}
\hline & Separation $(x / c)$ & Reattachment $(x / c)$ \\
\hline RANS & 0.660 & 1.200 \\
TA DES & 0.656 & 1.105 \\
Experiment & 0.680 & $1.110 \pm 0.003$ \\
\hline
\end{tabular}

we can see that both procedures accurately predict the onset of separation with the RANS procedure overpredicting the size of the separation bubble. The DES, however, properly predicts the reattachment point and the separation bubble size.

Figure 9 shows a stream trace velocity comparison. Here we can more easily see the flow reattachment locations and the effect the DES has on the flow. In Figure 9(b), we see that the streamline plot clearly shows that the RANS solution has a longer separation bubble than that observed experimentally in Figure 9(a). The time-averaged DES streamlines, Figure 9(c), show significant improvement for the predicted separation bubble length, with a 13.65\% shorter mean separation region compared to RANS and a mean reattachment length that is in good agreement with experimental measurements.

Time-averaged velocity profiles at $x / c=1.0,1.1,1.2$, and 1.3 , corresponding to locations within the separation region and slightly downstream of it, were also compared with experimental data. The peak reverse flow velocity predicted at $x / c=1.0$ in Figure 10(a) is close to the experimentally measured velocity and slightly lags in the region away from 


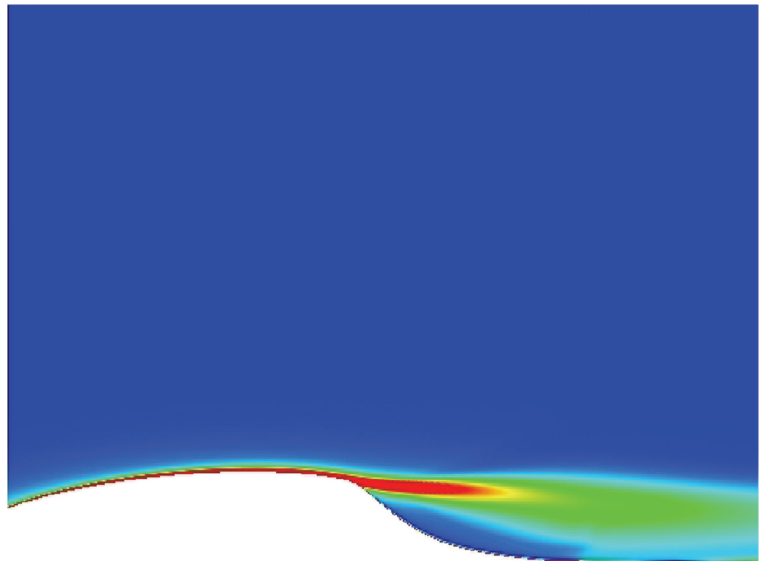

(a) RANS

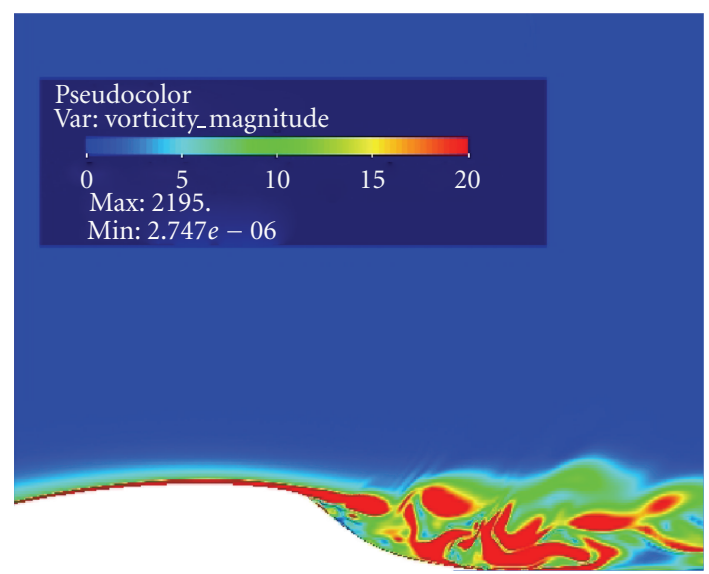

(b) Instantaneous DES

FIGURE 12: Spanwise averaged vorticity magnitude contours.

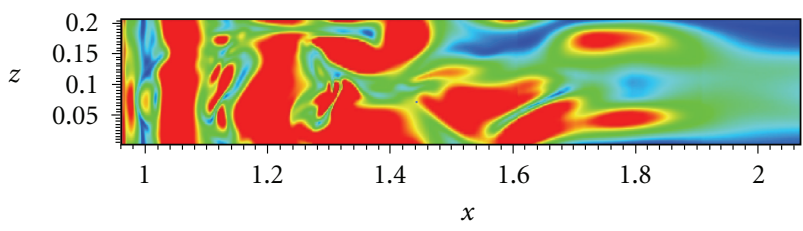

(a) $y=3.6576 \mathrm{~cm}$

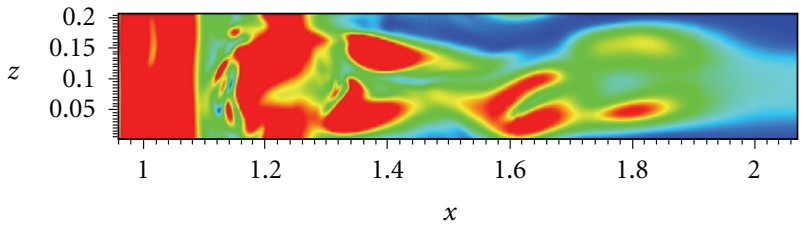

(b) $y=4.2672 \mathrm{~cm}$

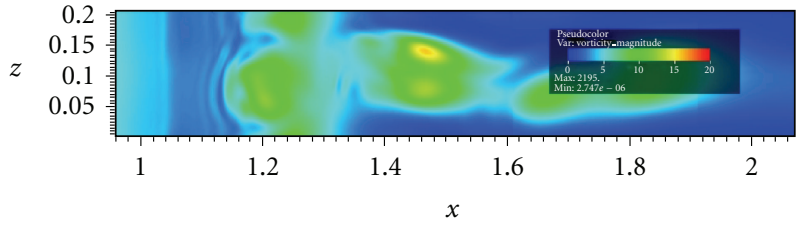

(c) $y=5.4864 \mathrm{~cm}$

FIGURE 13: Stream-slice vorticity magnitude contours at different distances away from wall.

the wall. Referring to the velocity profiles at $x / c=1.1$ and 1.2 in Figures 10(b) and 10(c), it can be seen that the experimentally measured flow reattachment point is at $x / c \approx$ 1.1, while MBFLO RANS and DES procedures predicted the reattachment point at $x / c \approx 1.2$ and 1.105 , respectively. In the near wall region of the flow, the DES procedure more accurately compares with the experimental velocity profiles at all four locations.

The DES and RANS profiles of the $v$-velocity component at $x / c=1.0$ and 1.1 in Figure 11 show good qualitative comparison at $x / c=1.0$ and 1.1 and match the experimentally measured values very well at $x / c=1.2$ and 1.3 . It should be noted that the $v$-component of the velocity is a magnitude smaller than the $u$-component and small changes can more readily be seen.

Figure 12 shows contour plots comparing the spanwise spatially averaged vorticity and for the RANS and instantaneous DES. The instantaneous vorticity contours in Figure 12(b) show a large range of resolved eddies consistent with large-eddy simulation treatment.
Instantaneous spanwise vorticity contour slices at $y=$ $3.6576,4.2672$, and $5.4864 \mathrm{~cm}$. normal to the wall are shown in Figure 13. Here, we can more easily see the threedimensionality that has formed in the wake of the wallmounted hump. Figure 14 presents instantaneous vorticity isosurfaces for the DES prediction. Here we can clearly see the separated shear layer downstream of the hump.

The detached-eddy simulation procedure, described by Bush and Mani [11], has been shown to be consistently more accurate than standard RANS. It predicts well the experimentally measured flow quantities such as pressure coefficient, surface skin friction, reattachment length, and mean velocity profiles. The RANS procedure predicted a delayed reattachment point, which indicates reduced turbulent mixing inside the separation region. Attempts at using higher-order numerical techniques, applied to RANS procedures $[1,3]$, have shown similar results. It should be noted that the DES procedure used a single constant model coefficient of 0.6 ; although additional computations using various coefficients would be essential to further examine 


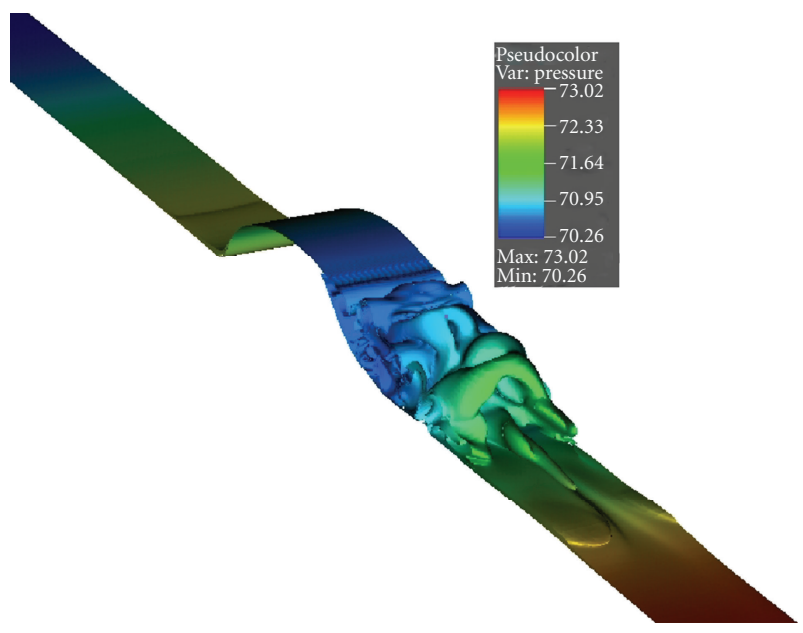

FIGURE 14: Vorticity isosurfaces (shaded by pressure) for DES prediction.

the DES procedure, it was beyond the scope of the current investigation.

\section{Conclusion}

A general Reynolds-averaged/detached-eddy simulation procedure was applied to the prediction of the flow over a wall-mounted hump. The initial results using the RANS and DES procedures compared well with experiment as well as other participants of NASA's 2004 Computational Fluid Dynamics Validation Workshop. Like other participants using RANS models, the onset of separation was accurately predicted and the reattachment point was overpredicted. The RANS procedure also overpredicted the mean pressure, skin friction, and velocity profiles in the separation zone. The DES procedure using the Bush and Mani model showed much better results. The three-dimensional structures resolved in the wake of the DES improved the local flow physics in the separation region and the predictions of the mean pressure distribution, skin friction, and streamwise velocity.

\section{Nomenclature}

E: Total energy

$e$ : Internal energy

$H$ : Total enthalpy

$h:$ Static enthalpy

$k$ : Turbulent kinetic energy

$p$ : Pressure

Pr : Prandtl number

$\operatorname{Pr}_{t}$ : Turbulent Prandtl number

$S_{i j}$ : Mean strain-rate tensor

$u_{i}$ : Velocity component

$V$ : Velocity magnitude

$\beta_{k, \omega}$ : Constants defined by $k-\omega$ equations

$\mu$ : Coefficient of viscosity

$\mu_{T}$ : Turbulent coefficient of viscosity $\omega: \quad$ Turbulent dissipation frequency

$\omega_{B}$ : DES filtered turbulent dissipation frequency

$\hat{\tau}_{i j}$ : Total shear stress tensor

$\tau_{i j}$ : Laminar shear stress tensor

$\tau_{i j}^{R}: \quad$ Reynolds shear stress tensor

$\rho:$ Density

Tu : $\quad$ Freestream turbulence level as \%

$l_{l e}$ : Eddy length scale

$\Delta: \quad$ DES filter width

$\sigma, \sigma^{*}$ : Constants defined by $k-\omega$ equations

$C_{\text {des }}$ : DES coefficients.

\section{Acknowledgments}

The authors would like to thank Dr. John Clark and the managers of the turbine branch at the Wright-Patterson Air Force Research Laboratory in Dayton, Ohio, for their support of this effort under contract 09-S590-0009-20-C1. Sandia National Laboratories is a multi-program laboratory managed and operated by Sandia Corporation, a wholly owned subsidiary of Lockheed Martin Corporation, for the U.S. Department of Energy's National Nuclear Security Administration under contract DE-AC04-94AL85000.

\section{References}

[1] P. E. Morgan, D. P. Rizzetta, and M. R. Visbal, "High-order numerical simulation of turbulent flow over a wall-mounted hump," AIAA Journal, vol. 44, no. 2, pp. 239-251, 2006.

[2] V. Krishnan, K. D. Squires, and J. R. Forsythe, "Prediction of separated flow characteristics over a hump," AIAA Journal, vol. 44, no. 2, pp. 252-262, 2006.

[3] P. Balakumar, "Computations of flow over a hump model using higher order method with turbulence modeling," in Proceedings of the 43rd AIAA Aerospace Sciences Meeting and Exhibit, Reno, Nev, USA, January 2005, AIAA Paper No. 20051270.

[4] D. M. Israel, D. Postl, and H. F. Fasel, "A flow simulation methodology for analysis of coherent structures and flow control," in Proceedings of the 2nd AIAA Flow Control Conference, 2004.

[5] S. J. Miller and P. A. Seitz, "The interaction between a fluidic actuator and main flow using SAS turbulence modeling," in Proceedings of the 3rd AIAA Flow Control Conference, San Francisco, Calif, USA, June 2006.

[6] F. R. Menter, "A scale-adaptive simulation model for turbulent flow predictions," in Proceedings of the 41st AIAA Aerospace Sciences Meeting, Reno, Nev, USA, 2003.

[7] D. C. Wilcox, Turbulence Modeling for CFD, DCW Industries, 3rd edition, 2006.

[8] D. C. Wilcox, "Reassessment of the scale-determining equation for advanced turbulence models," AIAA Journal, vol. 26, no. 11, pp. 1299-1310, 1988.

[9] J. Smagorinsky, "General circulation experiments with the primitive equations," Monthly Weather Review, vol. 91, no. 3, pp. 99-164, 1963.

[10] M. Strelets, "Detached eddy simulation of massively separated flows," in Proceedings of the 39th Aerospace Sciences Meeting and Exhibit, Reno, Nev, USA, January 2001, AIAA Paper No. 2001-879. 
[11] R. H. Bush and M. Mani, "A two-equation large eddy stress model for high sub-grid shear," in Proceedings of the 15th AIAA Computational Fluid Dynamics Conference, Anaheim, Calif, USA, June 2001, AIAA Paper No. 2001-2561.

[12] R. H. Ni, "Multiple-grid scheme for solving the euler equations," AIAA Journal, vol. 20, no. 11, pp. 1565-1571, 1982.

[13] J. F. Dannenhoffer, Grid adaptation for complex two-dimensional transonic flows [Ph.D. thesis], Massachusetts Institute of Technology, 1987.

[14] R. L. Davis, R.-H. Ni, and J. E. Carter, "Cascade viscous flow analysis using the Navier-Stokes equations," Journal of Propulsion and Power, vol. 3, no. 5, pp. 406-414, 1987.

[15] R. L. Davis, D. E. Hobbs, and H. D. Weingold, "Prediction of compressor cascade performance using a Navier-Stokes technique," Journal of Turbomachinery, vol. 110, no. 4, pp. 520-531, 1988.

[16] A. Jameson, "Time dependent calculations using multigrid with applications to unsteady flows past airfoils with wings," in Proceedings of the 10th Computational Fluid Dynamics Conference, p. 14, Honolulu, Hawaii, USA, June 1991, AIAA Paper No. 1991-1596.

[17] R. L. Davis and J. F. Dannenhoffer, "A detached-eddy simulation procedure targeted for design," in Proceedings of the 46th AIAA Aerospace Sciences Meeting and Exhibit, Reno, Nev, USA, January 2008, AIAA Paper No. 2008-534.

[18] A. J. Andrade, R. L. Davis, and M. A. Havstad, "A RANS/DES numerical procedure for axisymmetric flows with and without strong rotation," in Proceedings of the 46th AIAA Aerospace Sciences Meeting and Exhibit, Reno, Nev, USA, January 2008, AIAA Paper No. 2008-702.

[19] R. Bozinoski and R. L. Davis, "General three-dimensional, multi-block, parallel turbulent Navier-Stokes procedure," in Proceedings of the 46th AIAA Aerospace Sciences Meeting and Exhibit, Reno, Nev, USA, January 2008, AIAA Paper No. 2008756.

[20] A. Jameson, W. Schmidt, and E. Turkel, "Numerical solution of the Euler equations by finite volume methods using Runge Kutta time stepping schemes," in Proceedings of the 14th Fluid and Plasma Dynamics Conference, Palo Alto, Calif, USA, June 1981, AIAA Paper No. 1981-1259.

[21] W. Gropp, E. Lusk, and A. Skjellum, MPI: A Message-Passing Interface Standard, Scientific and Engineering Computation Series, The MIT Press, 1994.

[22] C. L. Rumsey, T. B. Gatski, W. L. Sellers, V. N. Vatsa, and S. A. Viken, "Summary of the 2004 computational fluid dynamics validation workshop on synthetic jets," AIAA Journal, vol. 44, no. 2, pp. 194-207, 2006.

[23] S. Šarić, S. Jakirlić, A. Djugum, and C. Tropea, "Computational analysis of locally forced flow over a wall-mounted hump at high-Re number," International Journal of Heat and Fluid Flow, vol. 27, no. 4, pp. 707-720, 2006.

[24] P. E. Morgan, D. P. Rizzetta, and M. R. Visbal, "Large-eddy simulation of separation control for flow over a wall-mounted hump," AIAA Journal, vol. 45, no. 11, pp. 2643-2660, 2007. 

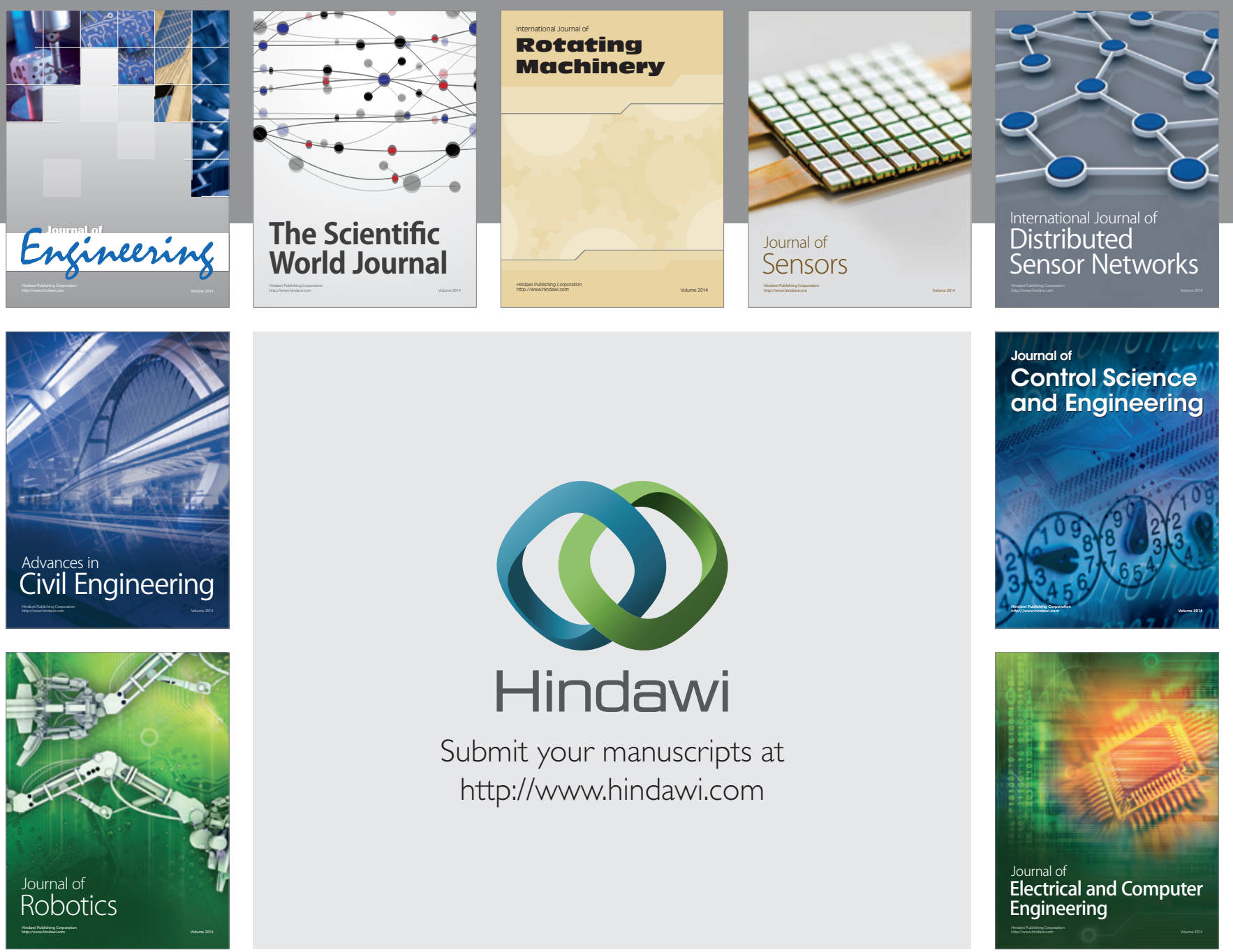

Submit your manuscripts at

http://www.hindawi.com
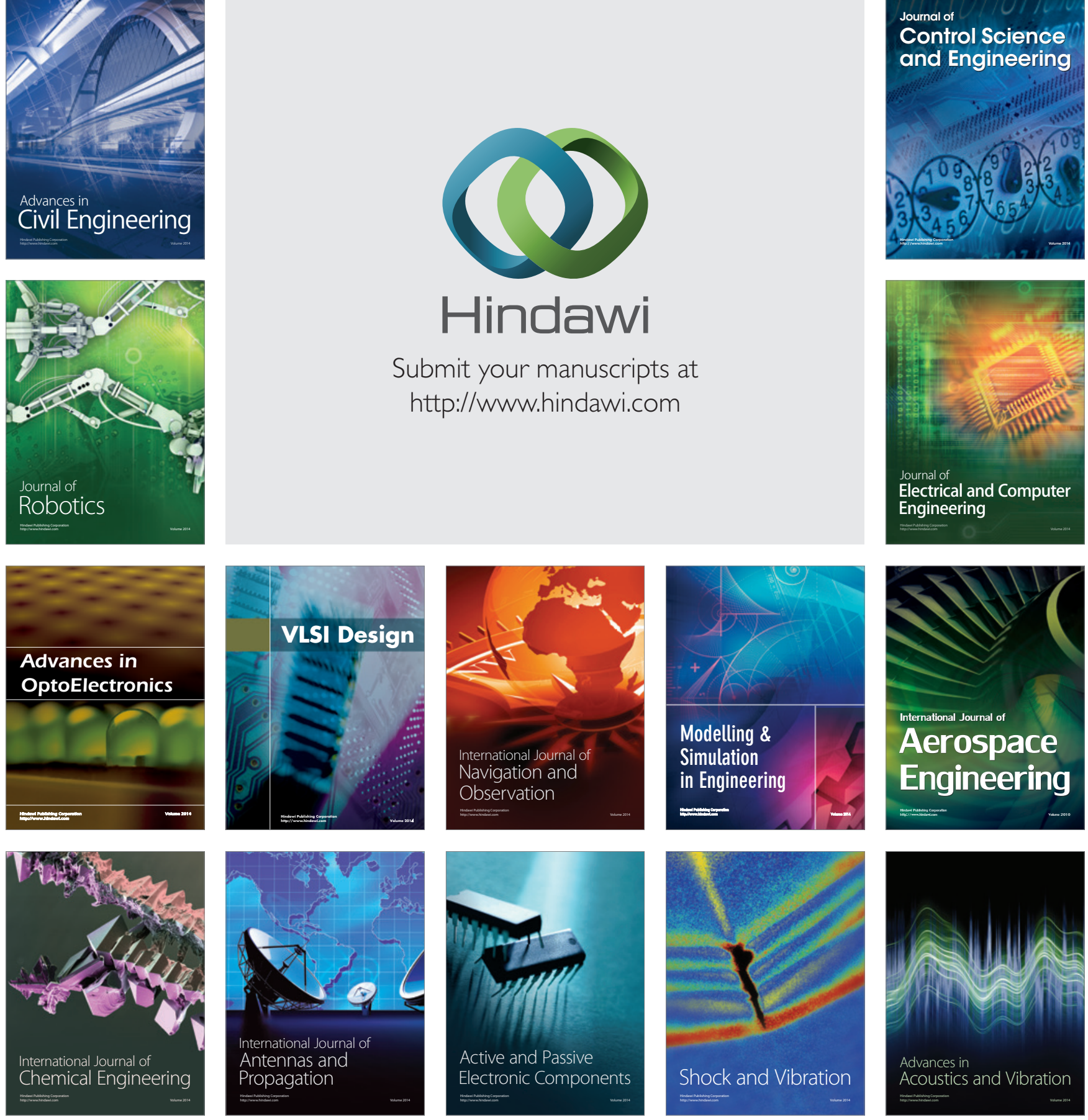